\title{
ON THE TEMPERATURE-DEPENDENT SURFACE STRUCTURE OF METALS
}

\author{
H.P. Bonzel aND U. Breuer \\ Institut für Grenzflächenforschung und Vakuumphysik Forschungszentrum Jülich \\ Postfach 1913, W-5170 Jülich, Germany . \\ (Received July 1, 1991; in revised form August 27, 1991)
}

\begin{abstract}
Recent experimental results based on X-ray photoelectron diftraction from a $\mathrm{Pb}(110)$ crystal are discussed in terms of surface roughening and surface melting. In comparison to other reports on $\mathrm{Pb}(110)$ surface roughening it is recognized that the correct determination of the roughening transition on (110) surfaces of fcc metals may be intrinsically difficult because of anisotropy. A novel technique for investigating the temperature-dependent surface free energy is described. The method involves the quasi-steady-state shape of periodic surface profiles on $A u(111)$ and $A u(100)$ single crystals. First results are reported for two crystallographic zones (111) $\langle 112\rangle$ and (100) $\langle 110\rangle$ of Au. These results illustrate the strong anisotropy of $\Delta \gamma / \gamma$ of $\sim 23 \%$ and $\sim 4 \%$ at $T=0 \mathrm{~K}$ for the (111) $\langle 112\rangle$ and $(100)\langle 110\rangle$ zones, respectively. The data suggest that the low-index surfaces of $A u(111)$ and (100) are not likely to roughen at $T \leq T_{M}$.
\end{abstract}

PACS numbers: $68.35 . \mathrm{Rh}, 68.35 . \mathrm{Md}, 79.60 . \mathrm{Cn}, 82.65 . \mathrm{Dp}$

\section{Introduction}

The temperature-dependent surface structure of metals is governed by the formation energies of defects and by interactions between defects. The latter are described by various approaches in statistical mechanics leading to the well-known concepts of a surface roughening phase transition $[1,2]$. The changes in surface structure can also be described phenomenologically by an orientation and temperature-dependent surface free energy, $\gamma(0, \varphi, T)$, which exhibits discontinuities (cusps) at low temperature characteristic of low-index orientations. These cusps vanish at the roughening temperature which means that low-index surfaces become energetically (and hence structurally) equal to vicinal surfaces: the formation energy of monatomic steps has become zero [3-5]. This process is in principle observable in the temperature-dependent change of the equilibrium shape of small metal particles $[6,7]$.

Another liigh temperature structural phase transition which has recently received a lot of attention is surface melting [8-12]. In this process long-range 
order is lost so that atoms are no longer occupying regular lattice sites, such as in a surface roughening transition. Surface melting is a phase transition of higher order, with the thickness of the molten layer growing logarithmically $[2,9,11]$. There is as yet no good understanding how these two processes, surface roughening and surface melting, can be linked together.

\section{Photoelectron diffraction of $\mathrm{Pb}(110)$}

Recent experiments of X-ray photoelectron diffraction (high kinetic energies) of a $\mathrm{Pb}(110)$ single crystal at various temperatures have illustrated the effect of surface melting and most likely of surface roughening as well [13-15]. Photoelectrons generated by $\mathrm{Mg} K_{\alpha}$ radiation are emitted from a $\mathrm{Pb}(110)$ single crystal surface and recorded by an angle-resolving spectrometer [13]. The emission pattern is quite anisotropic ( $50-60 \%)$ and characterized by forward-scattering enhancement peaks when the intensity is corrected for background and instrumental effects [16]. The amplitude of these enhancement peaks which occur along low-index crystallographic bulk directions is an excellent probe of short- and long-range (single crystal!) order within the probing depth of the electrons [17]. For a kinetic energy of $1400 \mathrm{eV}(\mathrm{Pb} 4 f)$ this probing depth is of the order of $30-50 \AA$, i.e. at least 17 layers of (110) orientation. Any disorder at the surface of $\mathrm{Pb}(110)$ extending over a couple of layers or penetrating deeper into the crystal is expected to diminish the intensity of the enhancement peak, in particular for a surface melting transition when atoms are no longer in lattice site positions.

An example of the temperature-dependent decrease in $4 f$ photoelectron intensity enhancement is illustrated in Fig. 1 for the [110] azimuth. Note in Fig. 1a that the relative $\Delta I(0)$ pattern is not changed as a function of temperature but that a general decrease in intensity occurs, especially at $T>500 \mathrm{~K}$. Figure $1 \mathrm{~b}$ shows a semi-log dependence of $\Delta I$ versus temperature plotted for three peaks at different polar angles. IIere the decrease is nearly linear up to about $500 \mathrm{~K}$ and nonlinear above. Since the intensity enhancement is due to forward scattering, the Debye-Waller factor is quite small $(\Delta k \approx 0)$, and therefore the slope in $\mathrm{d} \ln \Delta I / \mathrm{d} T$ at $T<500 \mathrm{~K}$ is small. Ilowever, the significant deviation from the extrapolated slope at $\sim 500 \mathrm{~K}$ calls for a drastic increase in the vibrational amplitude of surface atoms or a surface structural change at this temperature. In addition there is another small deviation near $360 \mathrm{~K}$ for off-normal emission peaks. This effect is only seen for this azimuth and not the [001] direction [13, 15]. It suggests a directionally dependent vibrational or structural effect starting near $360 \mathrm{~K}$.

At temperatures $>550 \mathrm{~K}$ there is a strong decrease in $\Delta I$ versus temperature. Clearly, this effect must be associated with surface melting and a complete loss of long-range order, as it has been found by ion scattering $[8,9,18]$ for the same surface. Our evaluation of the present data in those terms is then fully consistent with the ion scattering data [13]. On the other hand, the decrease in $\Delta I$ between 500 and $\sim 550 \mathrm{~K}$ cannot be solely attributed to surface melting, in particular because a different growth law for the disordered layer seems to prevail [13]. Also, the thickness of the disordered layer is about 1-2 layers only in this temperature range. The question arises whether the observed changes in intensity 

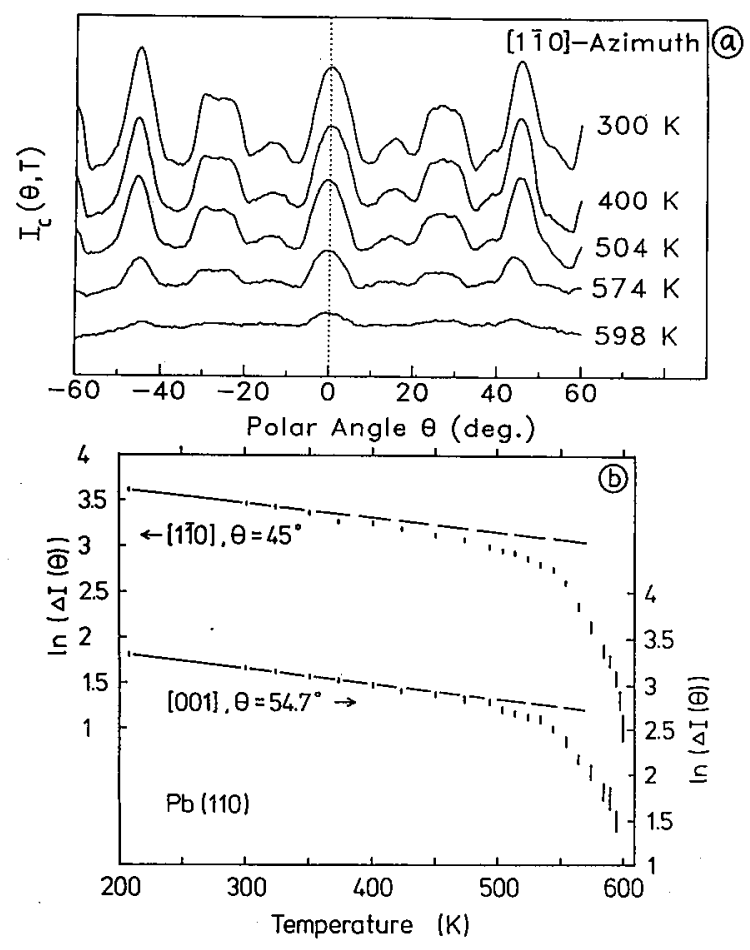

Fig. 1. Angle-resolved photoelectron intensities ( $\left.\mathrm{Pb} 4 f_{7 / 2}\right)$ from a $\mathrm{Pb}(110)$ crystal along the $[1 \overline{10}]$ azimuth at various temperatures. (a) Polar angle distributions. (b) Semi-log plot of enhancement peak intensities at certain polar angles versus temperature.

could not originate from a highly disordered surface, such as caused by surface roughening.

The process of surface roughening on a fcc(110) surface is expected to occur anisotropically $[3,5,15,19]$. The reason for this anisotropy is simply the existence of two types of surface steps parallel to the [110] and [001] directions. These steps have different energies. At the roughening temperature both step energies are to become zero but if one of the energies is considerably smaller than the other, steps of one kind will predominantly exist at temperatures much below $T_{R}$ of that surface [5]. Therefore the surface might be called rough if a technique sensitive to the lower energy step is used for surface structure analysis. A possibility for misjudging the roughening transition on a $\mathrm{fcc}(110)$ surface is given.

Investigations of surface roughening of $\mathrm{Pb}(110)$ quote roughening temperatures between 390 and 510 K [19-21]. Support for the higher temperature can be derived from the temperature-dependent surface free energy measured for $\mathrm{Pb}$ [7]. The anisotropy in $\gamma(0, T)$ for the (110) orientation exhibits the expected azimuthal dependence: a cusp is visible along the $\langle 100\rangle$ zone for $T \leq 548 \mathrm{~K}$ but not along the $\langle 110\rangle$ zone. The reason is the lowcr free cnergy of $\langle 110\rangle$ steps compared to $\langle 100\rangle$ 
steps [5]. The complete disappearance of the (110) cusp, i.e. along the $\langle 100\rangle$ zone, is noted at $573 \mathrm{~K}[7]$, i.e. a roughening temperature near $550 \mathrm{~K}$ might seem possible. This value is close to the temperature range of $500-550 \mathrm{~K}$ in Fig. $1 \mathrm{~b}$ where the drop in $\Delta I$ is noted for all peaks regardless of azimuth. We conclude that, because of the directional anisotropy of $\mathrm{Pb}(110)$, the true roughening temperature of that surface is more likely to be at $500-550 \mathrm{~K}$ than near $400 \mathrm{~K}$.

\section{Surface free encrgy anisotropy of $\mathrm{Au}$}

A new technique was used to study the anisotropy of the temperature-dependent surface free energy of Au along low-index zones. The technique is based on the quasi-steady-state shape of periodic profiles (wavelength $\sim 5-7 \mu \mathrm{m}$ ) which are etched into a low-index single crystal surface. These profiles are metastable because they decay with time at elevated temperature $[22,23]$ until a flat surface is obtained. However, during the decay they assume a quasi-steady-state shape which is largely dictated by the orientation-dependent surface free energy along the crystallographic zone covered by the profile $[24,25]$. The chemical potential (in one dimension) governing this process is [26]

$$
\mu(x, T)=\Omega\left\{\gamma(0, T)+\frac{\partial^{2} \gamma(\theta, T)}{\partial 0^{2}}\right\} K(x)
$$

where $\Omega$ is the atomic volume and $K(x)$ the one-dimensional curvature of the profile. The (polar) orientation 0 is a function of $x$. Assuming the profile decay to occur by surface self-diffusion, the shape of the profile can in principle be calculated if $\gamma(\theta, T)$ is known $[24,25]$. Of course, facet orientations correspond to discontinuities in $\partial^{2} \gamma / \partial 0^{2}$. On the other hand, $\gamma(\theta, T)$ itself is always defined, for faceted as well as non-faceted surfaces. The discontinuity in the derivatives of $\gamma(\theta, T)$ at the cusp was numerically approximated by a very local region of large curvature [24]. In this way facets on the profile shape and "cusps" in $\gamma(\theta, T)$ at low-index orientations could be correlated. The functional form of $\gamma(\theta, T)$ was obtained from a trial function with two adjustable parameters $[14,25]$.

Experimentally, the main task was to determine the profile shape at elevated temperature. Furthermore, the shape had to be of "steady-state" character, i.e. the rate of profile decay (decrease of amplitude) had always to be small compared to the rate of shape change. These features were evaluated from laser diffraction intensities generated by the profile [14, 27]. A comparison of measured and calculated intensities produced then the profile shape.

In a second step, the profile shape was computed from the diffusion dynamics including the chemical potential in Eq. (1). A trial function of $\gamma(\theta, T)$ was varied until the experimental profile shape could be matched. The input $\gamma(\theta, T)$ was then taken as the anisotropic surface free energy for the particular zone between the low-index and a high-index orientation that was expected to have the highest $\gamma$ for that zone.

Experiments were carried out for profiles on $\mathrm{Au}(111)$ and $\mathrm{Au}(100)$ crystals $[14,28]$. The surface cleanliness of these crystals was checked by Auger electron spectroscopy in all phases of the experiments. An example of a laser diffraction 
pattern from a $7 \mu \mathrm{m}$ profile on $\mathrm{Au}(111)$ at $T=1023 \mathrm{~K}$ is shown in Fig. 2a. This pattern is clearly untypical of a sinusoidal profile, for example [29]. The matched profile shape is illustrated in Fig. 2b. The amplitude is $0.135 \mu \mathrm{m}$ (in good
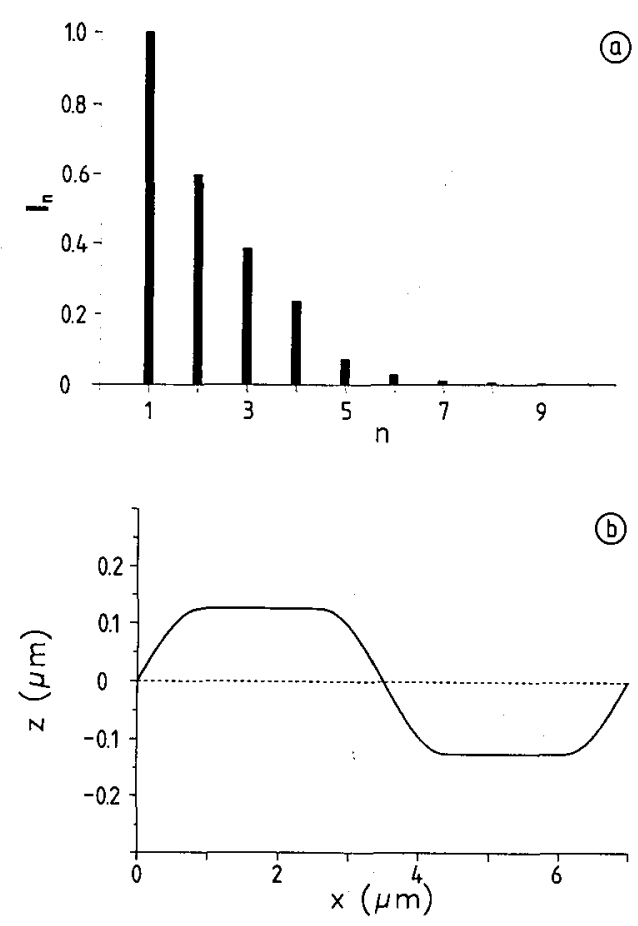

Fig. 2. Surface profiles on $A u(111)$ along the $\langle 112\rangle$ zone at $T=1023 \mathrm{~K}$ (groves of profile parallel to $\langle 112\rangle$ ). (a) Experimental (light) intensities versus diffracted order for a $7.0 \mu \mathrm{m}$ profile. (b) Corresponding profile shape calculated on the basis of an $8 \%$ anisotropy in $\Delta \gamma / \gamma$.

agreement with interference microscopy, ex situ) and the shape exhibits large flat regions parallel to the macroscopic (111) surface. This is expected because there is a deep minimum in $\gamma(0, \varphi)$ at the (111) orientation, equivalent to an anisotropy of $\sim 8 \%$ between the (111) and (210) orientations. This value is needed to obtain such a profile shape. The larger the "facet", the larger the total anisotropy for this zone.

The anisotropy $\Delta \gamma / \gamma(T)=\gamma_{\alpha} / \gamma_{\beta}-1$, where $\alpha$ is a high-index and $\beta$ a low-index orientation in the same crystallographic zone, was evaluated at various temperatures for the (111) $\langle 112\rangle$ and $(100)\langle 110\rangle$ zones. The $\alpha$ orientations were assumed to be (210) and (113), respectively [14]. A plot of $\Delta \gamma / \gamma(T)$ versus temperature is presented in Fig. 3 for the total temperature range between zero and $T_{M}$ of Au. Previously obtained data $[6,30]$ are also included for comparison. An 
interpolation function

$$
\frac{\Delta \gamma}{\gamma}(T)=\frac{e_{0 \alpha}-T s_{0 \alpha}}{e_{0 \beta}-T s_{0 \beta}}-1
$$

is used to describe the temperature dependence over the total range. Here $e_{0 \alpha}$,

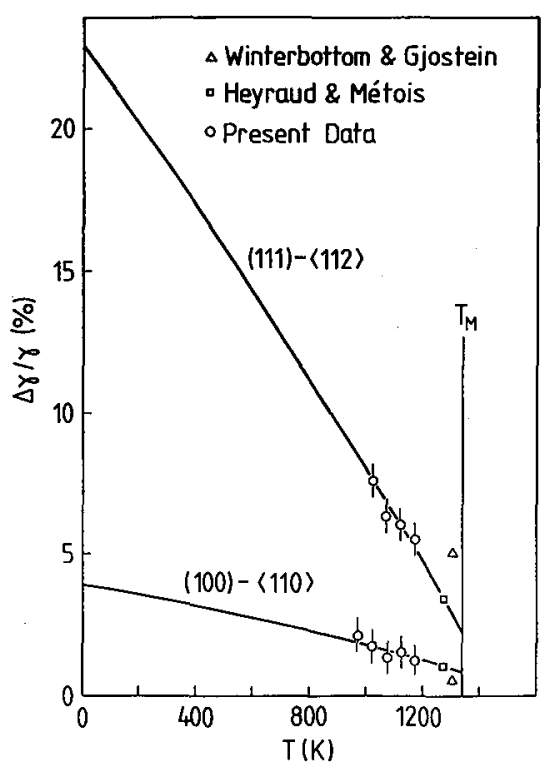

Fig. 3. Plot of relative surface energy anisotropy $\Delta \gamma / \gamma(T)$ versus temperature for $\mathrm{Au}(111)$ and $\mathrm{Au}(100)$ crystals along the $\langle 112\rangle$ and $\langle 110\rangle$ zones, respectively: Additional data are shown from Winterbottom and Gjostein [30] and Heyraud and Metois [6].

$e_{0 \beta}$ and $s_{0 \alpha}, s_{0 \beta}$ are surface internal energies and entropies for the $\alpha$ and $\beta$ orientations, respectively. All these quantities are assumed to be constant, i.e. changes with temperature due to roughening etc. are neglected. This assumptions is all right for the $\beta$ orientations but questionable for the $\alpha$ orientations. The main features of the functions in Fig. 3, however, are probably not dependent on small changes in $e_{0}$ and $s_{0}$ with temperature. These features are: firstly, the temperature dependence is much stronger for the (111) $\langle 112\rangle$ zone than for $(100)\langle 110\rangle$; this is due to the large anisotropy $\Delta \gamma / \gamma$ at $T=0$ for the (111) $\langle 112\rangle$ zone and the large difference in surface entropies of the (111) and (210) orientations. Secondly, the comparison of the present anisotropies $\Delta \gamma / \gamma$ at $T=0$ with recent theoretical values of $31.9 \%$ and $5.2 \%$ for $\mathrm{Au}(111)$ and $\mathrm{Au}(100)$ [3] is quite favourable. Thirdly, the general consistency between previous data for $\Delta \gamma / \gamma$ and the present values is very reasonable considering the difference in experimental approaches. Fourthly, the extrapolation of $\Delta \gamma / \gamma$ to $T=T_{M}$ shows that the low-index orientations (111) and (100) of $\mathrm{Au}$ are unlikely to undergo a roughening transition below $T_{M}$. 


\section{Conclusions}

The roughening temperature of a $\operatorname{lcc}(110)$ surface is difficult to determine because of the anisotropy in the free energies of $\langle 110\rangle$ and $\langle 001\rangle$ steps. The large range of roughening temperatures of $390-550 \mathrm{~K}$ quoted for $\mathrm{Pb}(110)$ are likely to be due to the phenomenon of anisotropic surface roughening. The likelihood of surface roughening of $\mathrm{Pb}(110)$ at $500-550 \mathrm{~K}$ suggests a close connection between this process and surface melting.

Temperature-dependent surface energy measurements of Au show anisotropies for the (111) $\langle 112\rangle$ and the (100) $\langle 110\rangle$ zones of $\sim 23$ and $\sim 4 \%$ at $0 \mathrm{~K}$, respectively. The (111) and (100) surfaces of Au are unlikely to roughen at $T \leq T_{M}$.

\section{References}

[1] J.D. Weeks, G.II. Gilmer, Adv. Chem. Phys. 40, Wiley, New York 1979, p. 157.

[2] H. van Beijeren, I. Nolden, in: Structure and Dynamics of Surfaces II. Topics in Current Physics, Vol. 43, Springer, Berlin 1987, p. 259.

[3] C. Jayaprakash, W.F. Saam, Phys. Rev. B 30, 3916 (1984); C. Jayaprakash, C. Rottman, W.F. Saam, Phys. Rev. B 30, 6549 (1984).

[4] C. Rottman, M. Wortis, Phys. Rev. B 29, 328 (1984).

[5] A. Trayanov, A.C. Levi, E. Tosatti, Surf. Sci. 233, 184 (1990).

[6] J.C. Heyraud, J.J. Metois, Acta Metall. Mater. 28, 1789 (1980).

[7] J.C. Heyraud, J.J. Metois, Surf. Sci. 128, 334 (1983).

[8] J.W.M. Frenken, J.F. van der Veen, Phys. Rev. Lett. 54, 134 (1985); J.W.M. Frenken, P.M.J. Maree, J.F, van der Veen, Phys. Rev. B 34, 7506 (1986).

[9] J.F. van der Veen, B. Pluis, A.W. Denier van der Gon, in: Chemistry and Physics of Solid Surfaces VII, Eds. R. Vanselow, R.F. Howe, Springer Series in Surface Science, Vol. 10, Springer, Berlin 1988, p. 455.

[10] D. Nenow, Prog. Crystal Growlh Charact. 9, 1985 (1984); A. Pavlovska, D. Nenow, Surf. Sci. 27, 211 (1971).

[11] R. Lipowsky, Speth, Phys. Rev. B 28, 3983 (1983).

[12] U. Breuer, H.P. Bonzel, K.C. Prince, R. Lipowsky, Surf. Sci. 223, 258 (1989); Phys. Rev. Lett. 62, 913 (1989); K.C. Prince, U. Breuer, H.P.Bonzel, Phys. Rev. Lett. 60, 1146 (1988).

[13] U. Breuer, O. Knauff, II.P. Bonzel, Phys. Rev. B 41, 10848 (1990); J. Vac. Sci. Technol. A 8, 2489 (1990).

[14] U. Breuer, Ph. D. Thesis, RWTH Aachen 1991.

[15] H.P. Bonzel, U. Breuer, M. Wortis, Surf. Sci, in print.

[16] C.S. Fadley, in: SynchrotrontKadiation Research: Advances in Surface Science, Ed. R.Z. Bachrach, Plenum Press, New York 1990; Prog. Surf. Sci. 16, 275 (1985).

[17] R. Trehan, C.S. Fadley, Phys. Rev. B 34, 6784 (1986).

[18] A.W. Denier van der Gon, R.J. Smith, J.M. Gay, D.J. O'Connor, J.F. van der Veen, Surf. Sci. 227, 143 (1990).

[19] A. Pavlovska, H. Steffen, E. Bauer, Surf. Sci. 234, 143 (1990).

[20] H.N. Yang, T.M. Lu, G.C. Wang, Phys. Rev. Lett. 63, 1621 (1989); Phys. Rev. B 43, 4714 (1991). 
[21] J.C. Heyraud, J.J. Metois, J. Cryst. Growth 82, 269 (1987).

[22] N.A. Gjostein, in: Adsorption et Croissance Crystalline, Coll. Intern. du C.N.R.S. Nr. 152. Editions C.N.R.S., Paris 1965, p. 97.

[23] W.W. Mullins, J. Appl. Phys. 28, 333 (1957); J. Appl. Phys. 30, 77 (1959).

[24] H.P. Bonzel, E. Preuss, B. Steffen, Appl. Phys. A 35, 1 (1984).

[25] H.P. Bonzel, E. Preuss, B. Steffen, Surf. Sci. 145, 20 (1984).

[26] C. Herring, in: Physics of Powder Metallurgy, Ed. W.E. Kingston, McGraw-Hill Book Comp., New York 1951, p. 143.

[27] K. Yamashita, H.P. Bonzel, H. Ibach, Appl. Phys. 25, 231 (1981).

[28] U. Breuer, H.P. Bonzel, to be published.

[29] H.P. Bonzel, N.A. Gjostein, J. Appl. Phys. 39, 3480 (1968).

[30] W.L. Winterbottom, N.A. Gjostein, Acta Metall. Mater. 14, 1041 (1966).

[31] D. Wolr, Surf. Sci. 226, 389 (1990). 